\title{
Reflets
}

Revue ontaroise d'intervention sociale et communautaire

\section{Conditions de possibilités des services en français en Ontario dans les domaines de la santé et des services sociaux : un enjeu pour les femmes}

\section{Lyne Bouchard et Linda Cardinal}

Volume 5, numéro 2, automne 1999

La santé des francophones de l’Ontario

URI : https://id.erudit.org/iderudit/026272ar

DOI : https://doi.org/10.7202/026272ar

Aller au sommaire du numéro

Éditeur(s)

Reflets : Revue ontaroise d'intervention sociale et communautaire

ISSN

1203-4576 (imprimé)

1712-8498 (numérique)

Découvrir la revue

Citer cet article

Bouchard, L. \& Cardinal, L. (1999). Conditions de possibilités des services en français en Ontario dans les domaines de la santé et des services sociaux : un enjeu pour les femmes. Reflets, 5(2), 109-122. https://doi.org/10.7202/026272ar

Tous droits réservés (C) Reflets : Revue ontaroise d'intervention sociale et communautaire, 1999
Ce document est protégé par la loi sur le droit d'auteur. L'utilisation des services d'Érudit (y compris la reproduction) est assujettie à sa politique d'utilisation que vous pouvez consulter en ligne. 


\section{Conditions de possibilités des services en français en Ontario dans les domaines de la santé et des services sociaux : un enjeu pour les femmes ${ }^{1}$}

\section{Lyne B ouchard}

La coopérative $C$ onvergence inc, $O$ ttawa

et Linda $C$ ardinal

professeure titulaire, D épartement descience politique, U niversité d' 0 ttawa

\section{Introduction}

Ce texte, conçu à l'intention des lectrices et lecteurs de la revue $R$ eflets, présente les faits saillants d'une recherche sur les conditions de possibilité des services en français en 0 ntario dans les domaines de la santé et des services sociaux². Les personnes ayant participé à l'équipe de recherche l'ont fait à titre de chercheures et de membres du R éseau des chercheures féministes de l'O ntario français (R CFO F).

L'objectif principal de la recherche est d'analyser les conditions de possibilité des services en français en 0 ntario et de proposer, dans les domaines de la santé et des services sociaux, des moyens propres à favoriser une plus grande prise en charge individuelle et collective des francophones et surtout, des femmes dans la 
mesure où elles sont habituellement exclues des processus de décisions. $\mathrm{N}$ ous avons accordé une attention particulière à I'interprétation que les actrices et acteurs en milieu francophone donnent à leur santé, aux services sociaux et de santé. U ne telle approche montre que les conditions de possibilité des services en français sont tributaires tant des perceptions des acteurs sociaux que des structures au sein desquelles ils se trouvent. En d'autres termes, les perceptionsinfluencent la structuration de l'action. La première étape a été de dégager un profil de la santé des francophones. Pour ce faire, nous avons procédé à l'analyse de données d'un sondage du gouvernement de l'O ntario, L'E nquête sur la santé en 0 ntario, réalisé en 1990.

U ne deuxième étape a été d'étudier les conditions de possibilités des services en français afin d'évaluer la capacité de prise en charge individuelle et collective des francophones et des femmes dans la mesure où la santé et les services sociaux constituent un enjeu important pour elles. De façon plus précise, nous avons étudié la dynamique institutionnelle au sein de laquelle les actrices et acteurs œuvrent à la mise en place des services en français. N ous avons aussi analysé le jeu des actrices et acteurs sur l'échiquier politique, en plus de nous intéresser au contexte politique et à la $L$ oi sur les services en français. $N$ ous avons aussi accordé une attention particulière aux dynamiques régionales, le cas échéant.

N ous avons procédé à une enquête de terrain dans quatre villes: $\mathrm{C}$ ornwall, $\mathrm{O}$ ttawa,T immins etToronto. En tout, nous avons rencontré 183 personnes œuvrant dans le domaine des services de santé et sociaux en français en 0 ntario. De plus, nous avons réalisé 24 entrevues de deux heures chacune avec des femmes choisies en fonction de critèresvisant à assurer la plus large diversité possible, à raison de six entrevues par ville.

$U$ ne troisième étape a été de faire des recommandations à la Table féministe francophone de concertation provinciale de I'O ntario (TFFCPO). Comme son nom l'indique, la Table féministe est une tribune dont le mandat principal est la concertation entre les groupes et l'action politique . Elle œuvre en 0 ntario depuis la fin des années 1980. Très tôt, à une époque où aucun groupe de femmes francophones travaillait véritablement 
sur cette question, laTable a choisi de privilégier le dossier de la santé comme un enjeu important pour les femmes. Ainsi, les membres de la Table ont marrainé cette étude pour avoir un portrait de la santé des femmes francophones de l'O ntario et connaître les dynamiques à l'œuvre dans le développement et la mise en place des services de santé et des services sociaux en français. En bout de piste, l'étude devait donner à la Table des moyens d'intervenir plus efficacement dansle domaine de la santé et des services sociaux.

\section{Un aperçu de la santé des femmes et des francophones : profil socio-sanitaire}

«A insi, nous constatons que les femmes francophones, comme ailleurs au $C$ anada et dans le monde, ont plus de problèmes de santé que les hommes »
Le profil socio-sanitaire des francophones de l'O ntario, esquissé en utilisant lesanalyses statistiques de l'E nquête sur la santé en 0 ntario, aura surtout permis de noter des tendances et de susciter un questionnement plutôt que de parvenir à des interprétations fermes ${ }^{3}$. Ainsi, nous constatons que les femmes francophones, comme ailleurs au $C$ anada et dansle monde, ont plus de problèmes de santé que les hommes. $0 \mathrm{n}$ remarque également que plus de femmes que d'hommes rapportent des jours alités ou passés dans l'incapacité de fonctionner normalement au cours des deux semaines précédant l'enquête. D ansl'ensemble, ce sont lesfemmes qui ont le plus recours aux services professionnels traitant de malaisespsychosociaux.

L'enquête montre que la perception de son état de santé varie selon les revenus du ménage et le niveau d'éducation des répondanteset répondants. Lesaînésfrancophones, et en particulier lesfemmes, dont le revenu et le niveau d'éducation sont inférieurs àla moyenne ontarienne, vivraient depuis longtemps les séquelles de ce déficit par une moindre qualité de vie. $N$ ous remarquons également que les francophones de 45 ans et plus auraient, comparativement aux anglophones, plus de problèmes de santé. D ans un autre ordre d'idées, on peut se demander pourquoi les 
«L es grandes variables explicatives qui traversent nos données sont l'âge et le sexe.»

« L es femmes ne dissodent pas la santé mentale de la santé physique. C'est pourquoi les femmes ont une conscience aiguë des rapports entre leurs conditions de vie et leur santé... » francophones des deux sexes présentent proportionnellement plus de problèmes musculo-squelettiques et plus d'accidents causant des blessures que les 0 ntariennes et les 0 ntariens de langue anglaise, en particulier les hommes.

Les grandes variables explicatives qui traversent nos données sont l'âge et le sexe. C ela dit, il semble que l'histoire de la communauté francophone et son développement économique sont déterminants pour comprendre la situation de nombreux aînés et nombreuses aînées francophones et leurs difficultés de santé. Par ailleurs, le profil de santé des femmes révèle des particularités qui pourraient s'expliquer par les rapports inégalitaires entre les sexes, en particulier, par une division inégalitaire du travail ou des revenus inférieurs.

\section{Le point de vue des femmes francophones}

U ne étape essentielle de notre recherche est consacrée à la réalisation de portraits qualitatifs de 24 femmes francophones vivant soit à Cornwall, $\mathrm{O}$ ttawa, Toronto ou Timmins. Pour ce faire, nous avons rencontré des femmes de divers milieux socioéconomiques, de diverses origines culturelles, d'âge et de statut civil. Leurs témoignages d'histoires et d'expériences personnelles, d'une extrême diversité, ont pourtant permis de dégager quelques grandes thématiques comme leurs conditions de vie, leur santé et les services en français.

Les femmes que nous avons rencontrées ont des conditions économiques modestes. C ela dit, leurs projets de vie, surtout s'ils prévoient l'éducation et la formation comme tremplin, connaissent un certain dynamisme. Elles ont aussi mentionné l'importance des réseaux familiaux et sociaux. Pour elles, cette importance se manifeste dans les relations entre une mère et ses enfants ou entre elles et leurs mères, et cela, peu importe que les femmes soient de souches anciennes ou récentes. Toutefois, l'isolement est une situation qui se vit au quotidien, ellesconnaissent une vie écartelée entre des tâches et des responsabilités multiples.

Les femmes ne dissocient pas la santé mentale de la santé physique. C'est pourquoi les femmes ont une conscience aiguë des rapports entre leurs conditions de vie et leur santé ou leur 
«L es femmes semblent avoir une approche globale de la santé privilégiant l'écoute de soi et l'information sur les traitements et les médicaments »
«Finalement, beaucoup de femmes ont développé des stratégies d' autosanté. » bien-être. Elles ont identifié des problèmes de santé divers: ulcères, manque d'énergie, détresse psychologique, etc. Certaines ont eu des cancers. D e façon générale, la santé pour les femmes est d'opter pour un mode de vie sain ou faire attention à ce que l'on mange, la recherche d'un certain équilibre et comprendre le lien étroit entre ses conditions de vie matérielle et psychologique et son état de santé. Les femmes semblent avoir une approche globale de la santé privilégiant l'écoute de soi et l'information sur les traitements et les médicaments. C ela dit, elleséprouvent souvent de la méfiance à l'égard des médicaments. Et, lorsque la maladie fait irruption dans leur vie, les femmes associent ces moments à des phénomènes de ruptures: incapacité de concilier toutes les tâches, accumulation de stress, rupture dans les liens familiaux et sociaux.

Ainsi, le sens que les femmes donnent à leur santé constitue un indice important des comportements qu'elles adopteront dans le milieu des services. Par exemple, certaines se disent en santé, même si elles ont eu un cancer. En effet, pour elles, le fait d'aller au travail le matin est un signe que tout va pour le mieux. D e plus, le rapport desfemmes avec le milieu des soins est l'expression d'une stratégie, c'est-à-dire un choix leur servant à mieux gérer les contraintes du quotidien afin de se redonner une marge de manoeuvre ou un certain contrôle sur leur vie $e^{4}$. Ainsi, les femmes ont recours au système des soins lorsqu'elles n'ont pas l'impression de pouvoir régler le problème elles mêmes, la famille et le réseau social étant à cet égard des espaces de soins pour les femmes. Finalement, beaucoup de femmes ont développé des stratégies d'auto-santé.

$D$ ans l'ensemble, les femmes ont beaucoup recours aux médecins de famille. $C$ eux-ci sont la plaque tournante des soins. Le médecin de famille est celui qui oriente les femmes vers les services spécialisés. De plus, elles ont recours à des services de counselling individuel ou de groupe dans les domaines de la violence, de la santé mentale et du counselling familial. Les groupes d'entraide ou de soutien sont aussi utilisés par les femmes. C ertaines ont recours à des services de médecine complémentaire ou holiste (naturopathes, homéopathes, chiropracticiens, etc.).

La majorité des femmes souhaitent recevoir des services de qualité, c'est-à-dire des services où le personnel est à l'écoute, 
"L'expression « bons services en français » cherche à désigner le fait que les femmes souhaitent généralement autant un bon service qu'un service en français... L es femmes souhaitent des services en français comme un choix auquel elles devraient avoir droit et non, comme un droit plus substantif. » compétent et professionnel. De plus, à leurs yeux, un service de qualité est un service respectueux des différences et accessible en fonction des horaires ou de l'endroit. II suppose aussi une certaine stabilité dans le personnel, notamment dansle $\mathrm{N}$ ord de la province où il n'est pas facile de retenir les spécialistes de la santé. Enfin, un service de qualité doit être polyval ent, c'est-à- dire qu'il doit offrir toute une gamme de services sous le même toit, comme c'est le cas de certains centres de santé communautairesfrancophones en O ntario, à C ornwall ou àToronto.

Lesfemmes demandent aussi desservices en français.Toutefois, force est de constater que ces derniers ne sont pas toujours disponibles, notamment dans le $\mathrm{N}$ ord de la province. D e plus, les femmes que nous avons rencontrées considèrent que leur priorité est un service qui réponde à leurs besoins, reste à savoir si le service en français fait partie de ces besoins.

L'expression «bons services en français » cherche à désigner le fait que les femmessouhaitent généralement autant un bon service qu'un service en français. La qualité du service est donc liée à la possibilité de l'obtenir en français. Les femmes souhaitent des services en français comme un choix auquel elles devraient avoir droit et non, comme un droit plus substantif. Elles considèrent souvent aussi que le médecin doit parler les deux langues officielles du pays. En ce sens, le service en français est lié au bilinguisme. C ela dit, le droit à un service en français est souvent contrecarré par l'absence de services ou par l'insuffisance des ressources en français, mais aussi par les conditions mêmes dans lesquelles se déroulel'acte médical et par leschoix desfemmespour desservices qu'elles considèrent de qualité.

\section{Les différences régionales}

$\mathrm{N}$ ous avons rencontré des femmes vivant dans quatre villes différentes: C ornwall, 0 ttawa, T immins, Toronto. Pour chacune de ces villes, les femmes ont so uvent soulignél'absence de services de santé en français, même à $O$ ttawa où ces derniers sont certes plus importants qu'àT immis ou àToronto. Elles dénoncent aussi les listes d'attentes et le roulement élevé de personnel qui vient interrompre le suivi médical. 


\section{Les conditions de possibilités des services de santé et des services sociaux en français}

\section{La dynamique institutionnelle}

En plus des entrevues en profondeur avec les femmes, nous avons rencontré des personnes œuvrant dans le milieu des services. C es professionnels et ces professionnelles de la santé et des services sociaux sont comme des personnes clés dans le développement des conditions de possibilités des services en français. C'est pourquoi nous avons cherché à savoir comment elles voient leur rôle dans le développement et la mise en œuvre des services en français? Ils et elles se voient au coeur de la dynamique de l'offre et de la demande et disent comprendre les besoins du milieu. En somme, elles et ils se perçoivent comme le lien entre le milieu et le service. $O r$, cette dynamique des services a semblé d'autant plus fragile qu'elle repose seulement sur lesépaules des personnes à la direction générale et des professionnels de la santé et des services sociaux. O $r$, la question qui se pose est la suivante : peuvent-ils assurer à eux seuls la défense, la mise en oeuvre et le développement des services?

$N$ ous avons aussi étudié la gestion de l'offre et de la demande afin de déterminer si le milieu des services tenait compte des besoins exprimés par les femmes. $M$ alheureusement, elles n'apparaissent pas dans la dynamique des services, à l'exception de quelques réflexions sur les femmes pauvres ou lorsque nous avons affaire à un service féministe. Par ailleurs, les parents sont

«A lors que les femmes sont éloignées du débat politique, les professionnels et les professionnelles de la santé sont surtout préoccupés par la dynamique politique.» souvent présents dans le discours des professionnels. À tel point qu'on se demande si l'intérêt démontré pour les parents ne pourrait pas se traduire par une plus grande attention à l'égard des femmes?

A lors que les femmes sont éloignées du débat politique, les professionnels et les professionnelles de la santé sont surtout préoccupés par la dynamique politique. Ils et elles ont sans cesse à justifier les services, à en montrer le besoin en fonction du 
«... il existe une

scission entre la dynamique linguistique et l'enjeu des soins de qualité. » critère linguistique plutôt qu'en fonction des femmes. A insi, ils et elles inscrivent leurs interventions dans une logique linguistique et autonomiste, tandis que les femmes se disent préoccuper par la qualité des rapports humains et leur quête de bien-être personnel.

Les conditions particulières qui président au développement et à la mise en place des services de santé et des services sociaux en français obligent les actrices et les acteurs à insister presque uniquement sur les enjeux linguistiques. De fait, il existe une scission entre la dynamique linguistique et l'enjeu des soins de qualité. $0 r$, cette dynamique des services n'est pas étrangère à l'avènement d'une catégorie de professionnels qui vise la mise en place d'un réseau parallèle de services sociaux et de santé, contrôlé par et pour les franco phones et ce, dansla foulée de l'adoption de la $L$ oi sur les services en français. $C$ ette revendication n'est pas sans rappeler les luttes dans le domaine de l'éducation pour l'obtention d'écoles françaises. II faut cependant souligner l'importance que les professionnels et les professionnelles du réseau de la santé, de concert avec les femmes, accordent à la nature communautaire des services en français. $C$ eux-ci semblent enracinés dans la réalité du milieu et ont pour but de répondre aux besoins culturels et linguistiques de la population. II y a donc convergence entre les groupes que nous avons rencontrés, dans la mesure où chacun, en dépit de leurs différences d'intérêts, souhaite un service communautaire et polyvalent.

Par contre, il nous semble que l'absence de dialogue entre les personnes œuvrant dans la santé et lesfemmes nuit aux conditions de possibilité d'une plus grande prise en charge dans le domaine de la santé et des services sociaux. Sans une convergence plus étroite des revendications desgroupesà la base et des professionnels, il nous semble que le souhait d'une prise en charge accrue risque de ne jamais advenir. C ertes, cette convergence existe déjà, notamment dans les services destinés aux femmes, mais aussi dans la représentation commune, souhaitée par les femmes et les professionnels, de services polyvalents, communautaires et en français. 
« L'idéal serait de favoriser le dialogue, d'envisager un partenariat entre les femmes et les professionnels de la santé et des services sodiaux. »

« L a population francophone et les femmes ne semblent pas véritablement associés au développement des services de santé et des services sodiaux en français. $C$ es derniers constituent des usagers plus que des acteurs ou des adrices »
L'idéal serait de favoriser le dialogue, d'envisager un partenariat entre les femmes et les professionnels de la santé et des services sociaux. Ce partenariat formulerait une vision de la santé où les femmes seraient reconnues comme des actrices pleines et entières et où s'accomplirait davantage un lien entre la santé et le social. Pour ce faire, il faut montrer que ce dialogue est indispensable à la prise en charge individuelle et collective des francophones par eux-mêmes.

\section{La dynamique politique}

La dynamique politique comprend à la fois le jeu des actrices et desacteurssur l'échiquier politique et lesstratégies qu'ils déploient afin de favoriser le développement des services de santé et des services sociaux en français. À ce chapitre, nous avons tenté de comprendre la perception que les acteurs que nous avons rencontrés ont de leur rôle dans le développement des services en français, de leur rapport avec le gouvernement et de la $L$ oi sur les services en français.

Au départ, il est important de souligner l'accent que certaines personnes ont mis sur l'activisme des groupes de femmes oeuvrant notamment dans le domaine de la violence. C ela dit, force est de constater que ce sont les directeurs généraux de services ainsi que les fonctionnaires qui jouent un rôle clé dans le développement et la mise en oeuvre des services en français. La population francophone et lesfemmesne semblent pas véritablement associés au développement des services de santé et des services sociaux en français. C es derniers constituent des usagers plus que des acteurs ou des actrices.

$\mathrm{N}$ ous avons identifié cinq types de stratégies que les actrices et acteurs mettent en branle sur l'échiquier politique en vue du développement des services en français dans les domaines de la santé et des services sociaux : des stratégies "personnalistes", paternalistes, dirigistes, partenariales et planificatrice.

Les stratégies «personnalistes » sont fondées sur le pouvoir d'influence des individus. Ainsi, selon plusieurs, le domaine de la santé exige beaucoup de tacts et d'astuces, des rapports personnalisées et un lobbying important. D ans ces intrigues de couloir, 
«... il semble que jusqu'à présent, le développement des services en français est dû à la volonté politique et à I'influence de certains individus et au pouvoir discrétionnaire de hauts foncionnaires » on fait appel à l'influence de personnes comme Bernard $G$ randmaître afin de faire débloquer des situations. U ne variante de ces dernières stratégies serait les stratégies paternalistes. Elles évoquent le pouvoir discrétionnaire attaché à certaines personnes occupant de hautes fonctions dans l'appareil politique (haut fonctionnaire, ministre, etc.). Bien que ce pouvoir existe de moins en moins, il a permis, par le passé, la création de services par voie de décret ministériel. Les stratégies dirigistes sont le fait, le plus souvent, de fonctionnaires et visent à orienter le développement des services en français dans les directions décidées en haut lieu. $D$ ans des milieux très anglophones, celles-ci sont parfois appropriées car seules les directives émanant des ministères peuvent faire avancer le dossier des services en français. Les stratégies partenariales sont associées à différents acteurs tant communautaires que gouvernementaux. $D$ ans ce cas, on accorde une grande importance à la collaboration entre les groupes dans le développement desservices, comme en ont témoigné les actrices et acteurs de la région de Cornwall. Q uant aux stratégies planificatrices, qui visent le développement de programmes et l'étude de besoins, ellesont notamment été utilisées par lesgroupes de femmes et les personnes oeuvrant au sein des ministères. C ela dit, nous constatons que les différentes stratégies sont plus le fait d'individus que de groupes.

En simplifiant, il semble que jusqu'à présent, le développement desservices en français est dû à la volonté politique et àl'influence de certains individus et au pouvoir discrétionnaire de hauts fonctionnaires. Ainsi, les stratégies « personnalistes » seraient les plus populaires. Pourtant, dans certaines régions, comme le $\mathrm{N}$ ord, où le milieu anglophone serait plus réfractaire aux services en français, les stratégies dirigistes ont connu un succès certain.

Par ailleurs, nous ne pouvons ignorer que les conditions de possibilités de services de santé en français ne sont pas étrangères à la dynamique politique qui avait cours à cette époque et notamment, de tous les débats sur l'unité canadienne. Aussi, il nous semble que les services en français sont fondés sur une philosophie et engagement politique imposés par le haut plutôt que par la base. C'est dans un tel contexte qu'en 1986, la L oi sur 
les services en français a été adoptée sous les leaderships de Bernard Grandmaitre et $D$ avid Peterson, entre aitres.

Plusieurs personnes nous ont dit que cette loi n'avait pas vraiment de mordant et qu'elle a surtout permis de rendre bilingues des services anglophones. À l'opposé, d'autres ont mentionné que la loi offrait l'opportunité de mettre en place de services parallèles en français. Force est de constater, en dépit des interprétations, que cette loi a inauguré une dynamique politique plus favorable au bilinguisme, à travers toute la question de la désignation, qu'au développement de servicesparallèlesen français. Les plus pessimistes ou réalistes considèrent également que l'arrivée au pouvoir du gouvernement conservateur de $\mathrm{M}$ ike $\mathrm{H}$ arrisa sonné le glas du développement des services parallèles en français dans les domaines de la santé et des services sociaux.

\section{Recommandations}

Au terme de notre étude, l'équipe de recherche a présenté cinq recommandations destinées à la Table et à ses groupes membres en vue de donner une portée concrète aux tendances notées et au questionnement soulevé dans ce rapport.

\section{Recommandation 1 : vision de la santé et des services sociaux}

La vision que les femmes ont de la santé et des services sociaux est absente du discours officiel en milieu francophone. Pourtant, les femmes savent identifier les conditions entraînant une rupture de leur état de santé et leurs multiples besoins face aux systèmes de santé et de services sociaux. N ous recommandons que laTable articule ces deux aspects de la problématique de la santé et des services sociaux en français en 0 ntario. En ce sens, laTable doit se faire promotrice d'une nouvelle vision de la santé et des services sociaux en milieu francophone. 


\section{Recommandation 2 : service idéal}

Les femmes veulent de bons services en français sauf que les contraintes du système sont telles qu'elles doivent parfoisfaire un choix entre de bons services et des services en français. 0 r, leur service idéal est un bon service en français, polyvalent et communautaire. $\mathrm{N}$ ous recommandons à la Table d'insérer cette dimension qualitative, l'idée du bon service en français, dans le débat sur la mise sur pied de services en français dansles domaines de la santé et des services sociaux.

\section{Recommandation 3 : service holiste}

Le système de santé et des services sociaux est un élément central au maintien de la santé des femmes. $0 r$, ce lien entre les femmes et le système de santé est perverti, puisqu'il se déroule sous le signe de logique parallèle. $D$ 'une part, le système de santé est mû par une logique de planification orchestrée par les professionnels. D 'autre part, lesfemmes demandent un système de soinscaractérisé par un personnel compétent, disponible, attentif, chaleureux, empathique et à l'écoute des clients. $N$ ous recommandons à la Table de favoriser l'émergence d'un dialogue entre ces deux logiques et de promouvoir tous les espaces de rencontre entre ces deux logiques.

\section{Recommandation 4 : engagement des femmes}

La venue des conservateurs au pouvoir a modifié le cadre politique dont sont tributaires les conditions de possibilité de services en français. Plus que jamais, il importe aux acteurs et aux actrices de s'engager dans le milieu municipal. D ans ces conditions, nous recommandonsà laTable d'articuler et de développer de nouvelles stratégiesd'action auprès du gouvernement provincial et despaliers régionaux ou locaux de municipalités.

\section{Recommandation 5 : diversité des femmes}

II sera important de poursuivre la recherche sur les multiples revendications des femmes, à l'image de leur diversité. À cet égard, lesfemmes vivant en milieu rural n'ont pasété un dessujets abordés 
dans cette étude, bien que nous ayons réalisé auprès de femmes du Sud-Est ontarien, certaines entrevues. Leur situation mériterait qu'on s'y attarde. C'est pourquoi nous recommandons à laTable d'approfondir avec les femmes, les enjeux spécifiques aux femmes francophones du Nord, aux femmes des milieux ruraux ou de milieux défavorisés et aux femmes francophones immigrantes. II faudrait voir aussi avec elles, les actions à privilégier en vue de favoriser leur plusgrande prise en charge individuelle et collective.

\section{Conclusion}

«D e plus, nous constatons un écart important entre les préoccupations des femmes francophones et celles des personnes cuvrant au développement et à la mise en place de services en français aux plans institutionnel et politique »
Les femmes que nous avons rencontrées ont une vision holiste du milieu de la santé et des services sociaux. C ela se traduit par des stratégies de soins combinant le réseau de soutien, le système de services socio- sanitaires, les servicesparallèles. Finalement, lesfemmes souhaitent de bons services en français. De plus, nous constatons un écart important entre les préoccupations des femmes francophones et celles des personnes œuvrant au développement et à la mise en place de services en français aux plans institutionnel et politique. Ainsi, les rapports entre le gouvernement et le milieu francophone demeurent fortement imprégnés par une dynamique de stratégiesindividuelles. Les conditions de possibilités des services en français sépendent donc actuellement del'existence d'un réseau «personnaliste » et de la volonté politique des gouvernements en place. S'il en est bien ainsi, le pouvoir de contrôle desfrancophones dans le domaine de la santé et des services sociaux est très fragile.

U ne meilleure prise en charge de la part des francophones dans le domaine des services de santé exigera un plus grand militantisme de la base. Les groupes de femmes œuvrant dans le domaine de la violence et des agressions à caractère sexuel l'illustrent bien. La nature de la dynamique politique nousamène à penser que l'avenir des services en français pourrait dépendre aussi de la capacité des femmes de développer un réseau d'acteurs collectifs partageant une vision de la santé qui s'articulerait autour du social et qui privilégierait des services de qualité en français. 
Enfin, une idée traverse l'ensemble de nos recommandations, celle qui fait des femmes, des actrices clés et incontournables dans I'utilisation des services par les familles francophones. C'est pourquoi les groupes membres de laTable féministe ont mandaté, lors de leur rencontre de février 1998, un comité pour voir à l'application des recommandations issues de la recherche. Le comité travaille actuellement à mettre en place un réseau pour la santé desfemmes. U n forum communautaire est prévu à cette fin à l'automne de 1999.

\section{Notes}

1. Vous pouvez vous procurer un sommaire de la recherche au numéro de courriel suivant : ucfo@web.net ou au (613) 241-2128.

2. La recherche a été réalisée sous l'égide de la Table féministe francophone de concertation provinciale de l'O ntario, en collaboration avec le R éseau des chercheuresféministes de l'O ntario français, et grâce à l'appui financier des ministères ontariens des Services sociaux et de la Santé. $\mathrm{N}$ ous tenons à souligner la contribution particulière des personnes participantes, à différents titres, à l'équipe de recherche : D yane A dam, C aroline Andrew, Françoise Boudreau, D enyse C ulligan, H élène D allaire, D iane Farmer, Gilles H uot, M ichelle K érisit, D enise Lemire, R éjean $\mathrm{N}$ adeau et Anne $\mathrm{R}$ ochon Ford.

3. Voir dans ce numéro le texte de Françoise Boudreau et Diane Farmer qui propose une analyse plus détaillée des données de l'étude sur le profil socio-sanitaire des francophones de la province.

4. Cette définition nous a été suggéré par Louise Blais, L'étrangère à nos portes.T hèse de doctorat, U niversité de M ontréal, 1994, p.27. 